\title{
Angular and Energy Dependences of Reflection Electron Energy Loss Spectra of Si*
}

\author{
H. Jin,$^{\dagger}$ H. Yoshikawa, H. Iwai, and S. Tanuma \\ Advanced surface chemical analysis group, National Institute for Materials \\ Science (NIMS), 1-2-1 Sengen, Tsukuba, Ibaraki 305-0047, Japan

\section{S. Tougaard} \\ Department of Physics and Chemistry, University of Southern Denmark, DK-5230 Odense M, Denmark
}

(Received 7 November 2008; Accepted 30 January 2009; Published 21 March 2009)

\begin{abstract}
Reflection electron energy loss spectroscopy (REELS) experiments at various emission angles were carried out using an inclined sample holder. The inclined sample holder can provide a wide range of emission angles to the surface normal $\left(15^{\circ}-75^{\circ}\right)$ by rotating the sample holder. The REELS spectra for Si were measured for electron beam energies from 500 to $4500 \mathrm{eV}$. The experimental $\lambda K(\Delta E)$ spectra of $\mathrm{Si}$, which are the product of the inelastic mean free path and the differential inverse inelastic mean free path, were obtained from the REELS spectra. It clearly showed the variation of relative contribution of bulk and surface loss in these series of the $\lambda K(\Delta E)$ spectra. We found that the bulk plasmon and surface plasmon contribution varied with the incident beam energies and emission angles. [DOI: 10.1380/ejssnt.2009.199]
\end{abstract}

Keywords: Reflection electron energy loss spectroscopy; Differential inverse inelastic mean free path; Bulk plasmon loss; Surface plasmon loss; $\mathrm{Si}$

\section{INTRODUCTION}

Reflection electron energy loss spectroscopy (REELS) is a useful technique in surface and interface analysis because the backscattered electrons provide information on the electron-solid inelastic interaction and can motivate the analysis of nano structure using medium primary energy $[1,2]$. When the energetic electron is transported into a solid, the incident electron can be inelastically scattered through interaction with either outer- or inner-shell atomic electrons. The REELS is a complicated combination of separate physical phenomena including bulk and surface plasmon excitation, interband transition, multiple scattering and momentum transfer. Plasmon losses correspond to a collective oscillation of the valence electrons and their energies are related to the density of valence electrons and sensitive to changes in the band structure. Extracting differential inverse inelastic mean free path (DIMFP) from quantitative analysis of REELS can also give us direct information on the electronic properties of the dielectrics [3]. DIMFP of electrons is an important bulk-material-parameter for surface electron spectroscopy analysis $[4,5]$. It determines quantities like the inelastic mean free path (IMFP) [6] Besides, DIMFP gives us the energy loss function $\operatorname{Im}[-1 / \hat{\varepsilon}][7]$, which is directly connected to optical constants $\hat{\varepsilon}$.

The purpose of this study is to obtain the variation of the bulk and surface loss contribution with the primary electron energies and emission angles of REELS spectra for Si. We also obtained the experimental DIMFP from series of REELS spectra directly. These series of DIMFP spectra clearly showed the variation of relative contribution of bulk and surface loss dependent on the emission angles and primary beam energies. It indicates that not a small number of REELS spectra but series of REELS

\footnotetext{
* This paper was presented at International Symposium on Surface Science and Nanotechnology (ISSS-5), Waseda University, Japan, 9-13 November, 2008.

${ }^{\dagger}$ Corresponding author: JIN.Hua@nims.go.jp
}

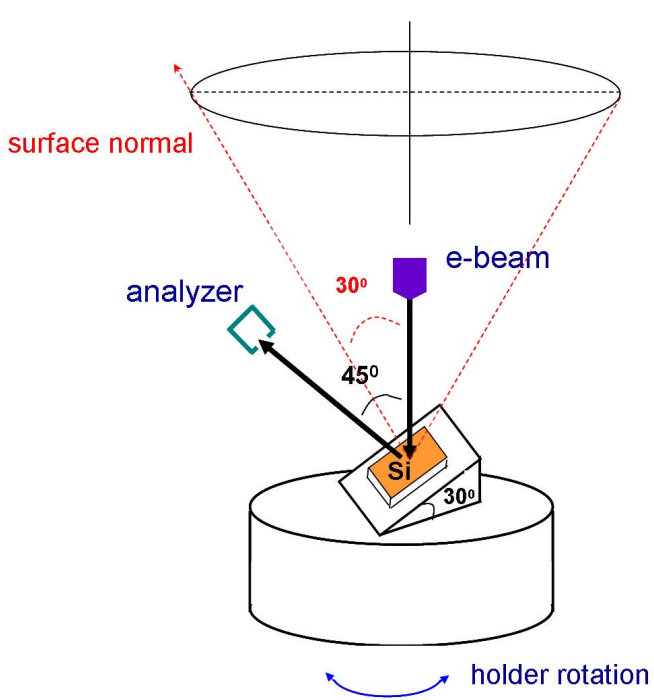

FIG. 1: Diagram of geometry for the reflection electron energy loss spectroscopy experiments.

spectra enable to reveal the surface and bulk loss components.

Separation of bulk and surface loss contribution in the experimental DIMFP can give us the above-mentioned valuable information on the electronic structure and the knowledge of surface excitation described by the surface excitation parameters (SEP) [8]. In a further study, we will use principal component analysis method [9] to derive the surface and bulk loss contribution precisely $[10,11]$.

\section{EXPERIMENTAL}

The REELS spectra were measured using Ulvac-Phi model 5500 electron spectrometer equipped with a concentric hemispherical analyzer. Measurements were performed for electron beam energies from 500 to $4500 \mathrm{eV}$. The spectra were measured in the constant analyzer en- 
ergy mode with pass energy of $23.5 \mathrm{eV}$ and at energy increments of $0.1 \mathrm{eV}$. REELS experiments with various emission angles were carried out using an inclined sample holder. Figure 1 shows the diagram for the geometry of the REELS experiments. The Si sample was mounted on a sample holder which was inclined by $30^{\circ}$. This setup can provide a wide range of emission angles to the surface normal $\left(15^{\circ}-75^{\circ}\right)$ when rotating the sample holder. The electron-gun axis is in the vertical direction. This gives a constant incidence angle of $30^{\circ}$ with respect to the surface normal. The angle between the axis of the electron-gun and the analyzer is $45^{\circ}$. We obtained a series of spectra with different emission angles for fixed primary beam energy. The Si sample was sputtered for 5 minutes using 2 $\mathrm{keV} \mathrm{Ar}^{+}$sputtering for cleaning and removing the surface contamination. The cleanness of the sample was checked by Auger electron spectroscopy. The base pressure in the analysis chamber was approximately $<2 \times 10^{-9}$ Torr during the spectra measurement.

\section{RESULTS AND DISCUSSION}

Before measuring the REELS spectra, it should be checked that the spectrometer is in the condition of the linearity response of the analyzer. As the level of signals in the elastic peak and inelastic parts of the spectrum are rather different, it is important that the analyzer response is linear and does not saturate in the energy region of the elastic peak [12]. Here we measured the REELS spectra for primary energy $3000 \mathrm{eV}$ several times by increasing the electron beam from $10 \mathrm{nA}$ to $280 \mathrm{nA}$ in step of $10 \mathrm{nA}$ for the first twelve spectra. The increment in the beam current over $120 \mathrm{nA}$ is different from those below $120 \mathrm{nA}$. Figure 2 shows the results to check the linearity of analyzer response. We plotted the relation of elastic peak areas with beam currents. We can see that there is a monotonic increase of elastic peak areas with increasing beam current. We have also drawn a straight line passing through the zero point corresponding to the ideal linearity response behavior. Comparing with the straight line, we divided the beam currents into three regions. The first region with beam current from about of 0 to $138 \mathrm{nA}$, the response of the analyzer is linear and in addition with these currents we get a good signal-to-noise spectrum with a sharp elastic peak. The beam current region of 138-240 $\mathrm{nA}$, where the measured intensities are below the ideal straight line, is the region where we get saturation of the analyzer. Here we obtain spectrum with broadened elastic. It means the saturation of the spectrometer in the energy region of the elastic peak. In the third region with beam currents $>240 \mathrm{nA}$, the elastic peak is split because the signal counts are larger than the digital detection level of the spectrometer. The linearity requirement sets a limit on the maximum beam current that can be used. If the spectrometer is found to be saturated, one should lower the primary beam current to the normal range where one gets the spectrum with a good signal-to-noise ratio and a sharp elastic peak. During our spectra measurement, the intensity of elastic peak was in million counts/second magnitudes for ideal spectra, which was an order of magnitude smaller than the saturation intensity.

Figure 3 shows the reflection electron energy loss spec-

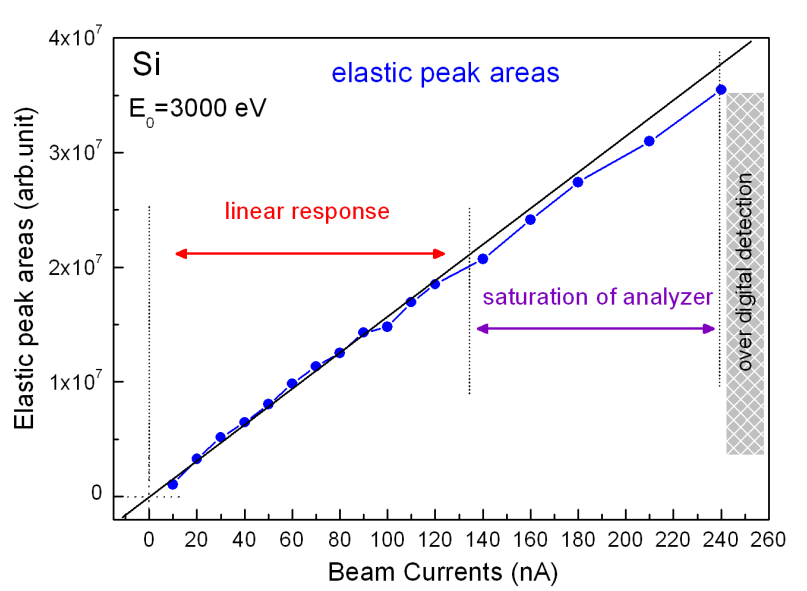

FIG. 2: The results to check the linearity of analyzer response.

tra of Si at primary electron energies of 500, 1000, 2000, 3000,4000 and $4500 \mathrm{eV}$ at $15^{\circ}$ emission angle. The spectra were normalized at the elastic peak area. In REELS spectra, incident electrons are elastically or inelastically scattered from the sample. This process results in the excitation of plasmons, interband transitions and elastically and inelastically scattered electrons. Collective excitations and electronic transition are considered to characterize the REELS spectra. Surface and bulk plasmons were identified as the peaks at about 11 and $16.8 \mathrm{eV}$ for $\mathrm{Si}$ [13]. The surface plasmon peak was very weak for spectra at emission angle of $15^{\circ}$ and appeared as a broad weak shoulder. As the primary energies increase, the intensity of the bulk plasmon peak relative to that of the surface plasmon peak became larger. Besides, the numbers of multiple bulk plasmon $(n \hbar \omega)$ increased. As one might expect, the interval between these multiple bulk plasmons was almost identical. The REELS spectrum for $500 \mathrm{eV}$ showed three multiple plasmon peaks whereas five multiple plasmon peaks can be distinguished for the spectrum at $4500 \mathrm{eV}$. The figure clearly demonstrates the energy dependence of REELS spectra from a Si sample.

Figure 4 shows the comparison of REELS spectra at various emission angles for primary electrons of energy 500, 1000, 2000, 3000, 4000 and $4500 \mathrm{eV}$. All the spectra were normalized at the elastic peak. The bulk plasmon peak at $16.8 \mathrm{eV}$ and the surface plasmon at about $11 \mathrm{eV}$ for $\mathrm{Si}$ were clearly recognized for spectra at low primary energies and high emission angle. For each series of spectra at a fixed primary electron beam energy, the surface plasmon increased in intensity relative to the bulk plasmon in particular for emission angles higher than $56^{\circ}$. These series of spectra showed the gradual variation of bulk and surface plasmon peaks with the primary electron energies and emission angles.

A series of the experimental $\lambda K(\Delta E)$ spectra calculated from the corresponding REELS spectra at various emission angles was shown in Fig. 5. The $\lambda K(\Delta E)$ spectrum was obtained using QUASES-XS-REELS software developed by Tougaard $[12,14] \lambda K(\Delta E)$ means the product of the inelastic mean free path and DIMFP. The DIMFP $K(E, \Delta)$ is the probability that an electron of energy $E$ shall lose energy $\Delta E$ per unit energy loss and per unit path length traveled in the solid. Since multiple 


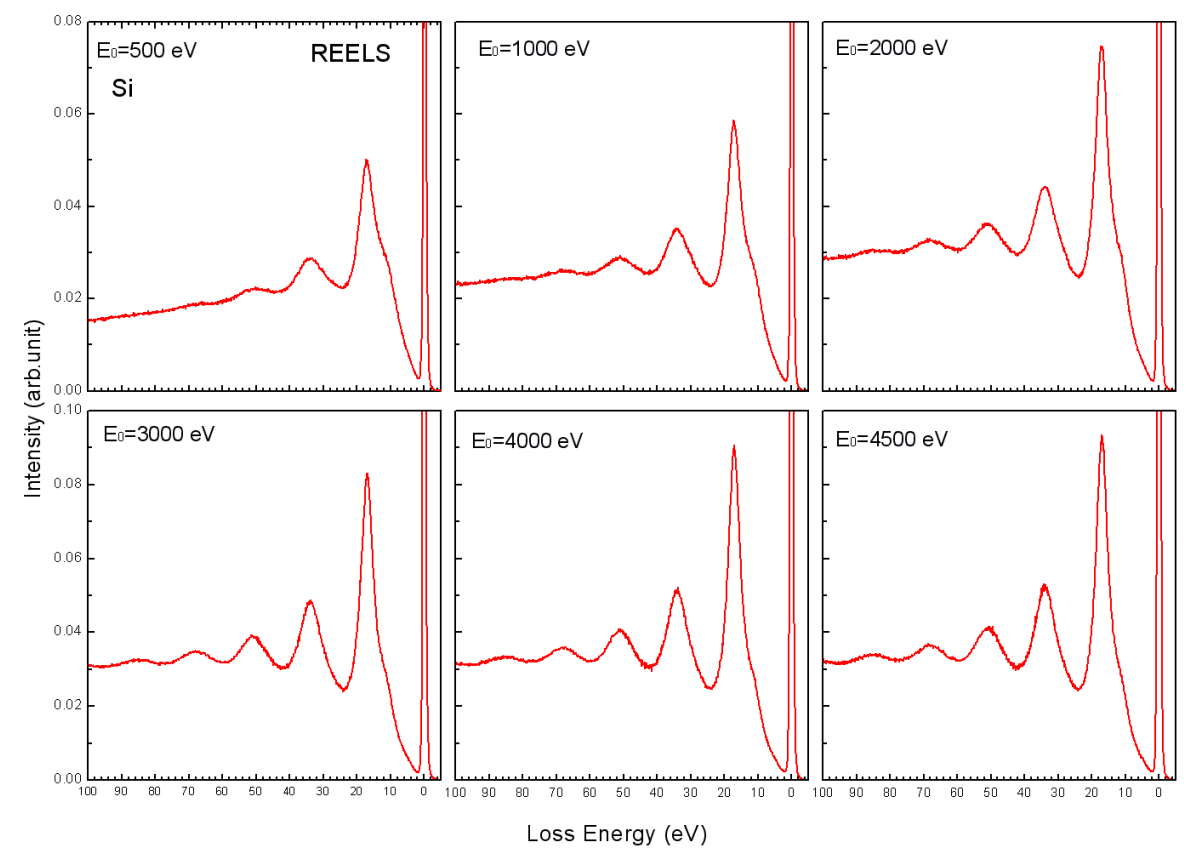

FIG. 3: The reflection electron energy loss spectra of Si at primary electron energies of 500, 1000, 2000, 3000, 4000 and 4500 $\mathrm{eV}$ at $15^{\circ}$ emission angle.

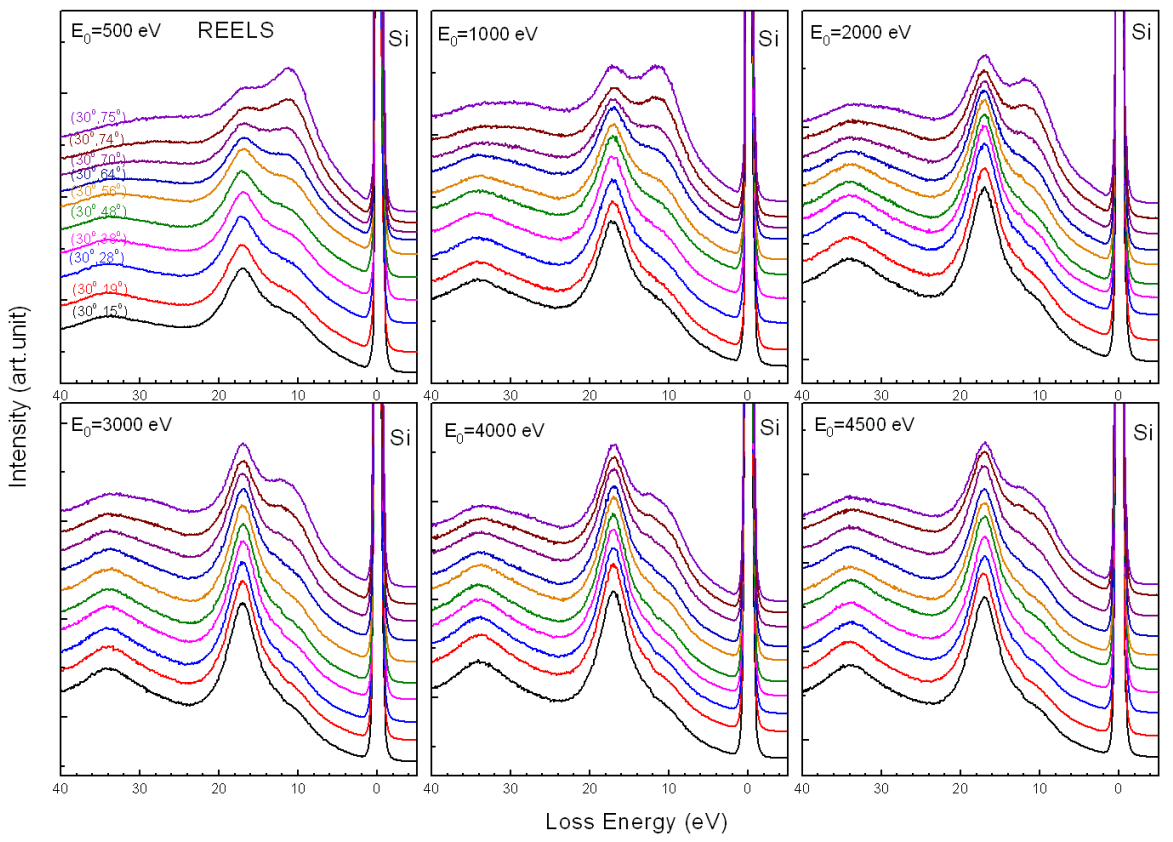

FIG. 4: Comparison of reflection electron energy loss spectra of Si at various emission angles for primary electrons of energy 500, 1000, 2000, 3000, 4000 and $4500 \mathrm{eV}$.

scattering features were removed in the $\lambda K(\Delta E)$ curve, it was easier to interpret the electronic excitations. The bulk and surface plasmon are now clearly identified. There was an obvious surface loss peak at about $11 \mathrm{eV}$ for spectra at $500 \mathrm{eV}$ and for higher emission angles. At higher primary energy and low emission angle, the contribution of bulk loss was significant and a little surface loss feature gradually became visible with increasing emission angle. These $\lambda K(\Delta E)$ spectra clearly showed the variation of bulk and surface loss contribution with the changing of primary electron energies and emission angles. The negative part of the spectra appeared at $\sim \hbar \omega_{s}+\hbar \omega_{p}$ and is due to deficiencies of the algorithm to correct for the mixture of surface and bulk plasmon scattering from an experimental REELS experiment $[5,15,16]$ Here the parameter $\hbar \omega_{s}$ is the surface plasmon energy and $\hbar \omega_{p}$ is the bulk plasmon energy. This effect is only seen for solid with a sharp intense plasmon structure (like in $\mathrm{Al}$ and $\mathrm{Si}$ ). The 


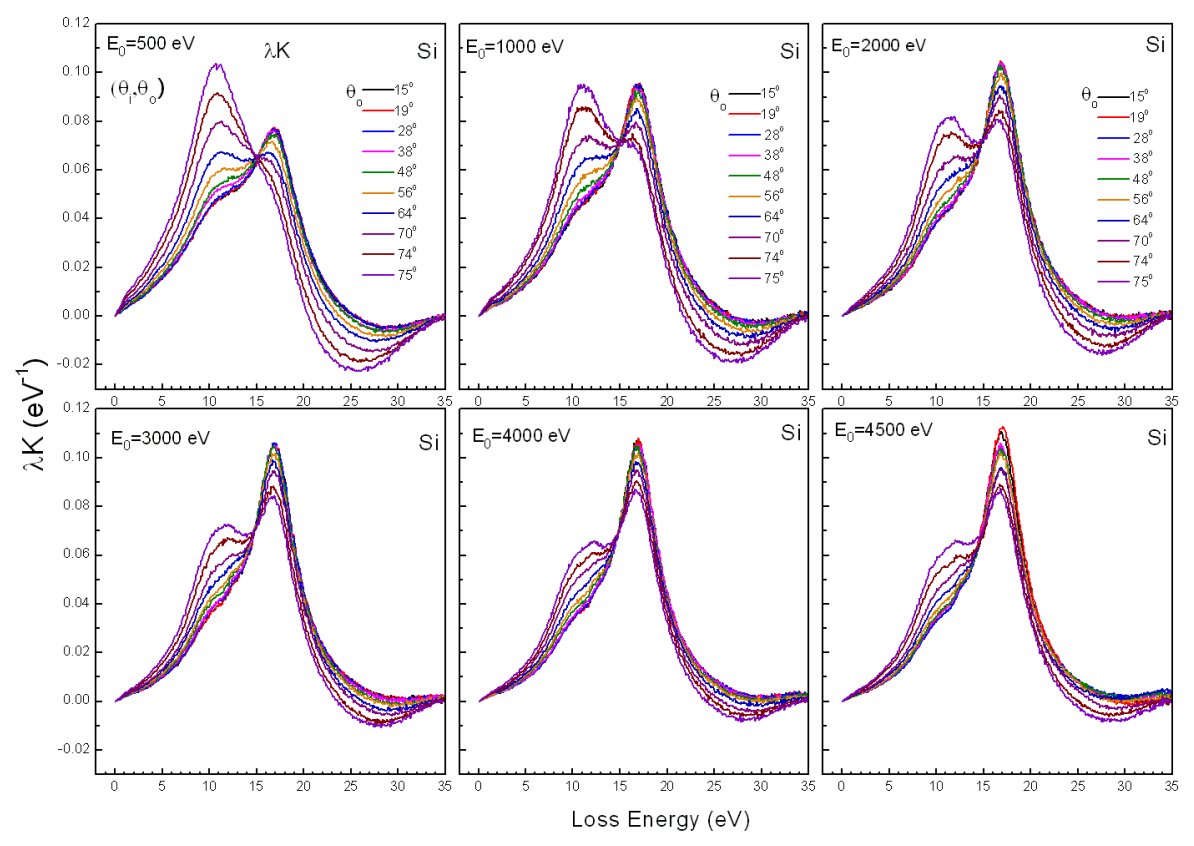

FIG. 5: Series of the experimental $\lambda K(\Delta E)$ spectra obtained from the corresponding REELS spectra in Fig. 4 at various emission angles. $\theta_{0}$ is the electron emission angle with respect to the surface normal.

negative parts are ascribed to the differences of estimated number of multiple scattering events in the Tougaard's algorithm and the experimental situation. Therefore, the relative intensities in the various multiple surface- and bulk- plasmon excitation peaks will be slightly different from the real experimental situation [16]. The negative parts of the spectra became more pronounced with decreasing primary beam energy and at higher emission angles. For spectra at primary energies higher than 2000 $\mathrm{eV}$, only a small negative parts of $\lambda K(\Delta E)$ appeared at high emission angle.

\section{CONCLUSIONS}

We measured the REELS spectra of Si with the variation of primary energies and emission angles. We can conveniently obtain various emission angles by rotating an inclined sample holder. In the REELS spectra, the multiple bulk plasmon peaks appeared and became more pronounced at higher primary beam energy. The surface loss contribution increased at high emission angles. From these series of REELS spectra, we determined the experimental $\lambda K(\Delta E)$ spectra. The variation of the relative contribution of bulk and surface plasmon were clearly observed in these series of spectra. Our analysis was based on the changing of the primary beam energies and emission angles. The results showed the variation of relative contribution of bulk and surface loss of REELS spectra dependent on the emission angle and primary electron energy in the series of spectra. In a further study, we will use factor analysis method to derive the surface and bulk loss contribution precisely and estimate some important parameters, such as optical constants and the surface excitation parameter.
[1] R. F. Egerton, Electron Energy-loss Spectroscopy in the Electron Microscopy, 2nd. ed. (Plenum, NewYork, 1996).

[2] H. Jin, S. K. Oh, H. J. Kang, and M.-H. Cho, Appl. Phys. Lett. 89, 122901 (2006).

[3] H. Jin, S. K. Oh, H. J. Kang, and S. Tougaard, J. Appl. Phys. 100, 083713 (2006).

[4] F. Yubero, J. M. Sanz, B. Ramskov, and S. Tougaard, Phys. Rev. B 53, 9719 (1996).

[5] F. Yubero, D. Fujita, B. Ramskov, and S. Tougaard, Phys. Rev. B 53, 9728 (1996).

[6] F. Yubero, J. M. Sanz, J. F. Trigo, E. Elizalde, and S. Tougaard, Surf. Interface. Anal 22, 124 (1994).

[7] J. C. Ingram, K. W. Nebesny, and J. E. Pemberton, Appl. Surf. Sci. 44, 279 (1990).

[8] G. Gergely, M. Menyhard, S. Gurban, A. Sulyok, J. Toth, D. Varga, and S. Tougaard, Surf. Interface Anal. 33, 410 (2002).
[9] A. M. Konovalov, Yu. M. Krynko, Y. S. Musatenko, and M. G. Nakhodkin, J. Electron Spectrosc. Relat. Phenom. 133, 27 (2003).

[10] P. Prieto, F. Yubero, E. Elizalde, and J. M. Sanz, J. Vac. Sci. Technol. A 14, 3181 (1996).

[11] N. Pauly and S. Tougaard, Surf. Sci. 602, 1974 (2008).

[12] S. Tougaard and J. Kraaer, Phys. Rev. B 43, 1651 (1991).

[13] F. Yubero, S. Tougaard, E. Elizalde, and J. M. Sanz, Surf. Interface Anal. 20, 719 (1993).

[14] http://www.quases.com

[15] S. Hajati, O. Romanyuk, J. Zemek, and S. Tougaard, Phys. Rev. B 77, 155403 (2008); ibid. B 77, 249904 (2008).

[16] S. Tougaard and I. Chorkendorff, Phys. Rev. B 35, 6570 (1987). 